Published in final edited form as:

Crit Rev Oncol Hematol. 2018 May ; 125: 1-11. doi:10.1016/j.critrevonc.2018.02.008.

\title{
Efficacy and Toxicity Profile of Carfilzomib Based Regimens for Treatment of Multiple Myeloma: A Systematic Review
}

\author{
Adeela Mushtaq $^{a, 1}$, Vikas Kapoor ${ }^{a, 1}$, Azka Latif $^{a, 1}$, Ahmad Iftikhar $^{1}$, Umar Zahid $^{1,2}$, Ali \\ McBride $^{3}$, Ivo Abraham ${ }^{4}$, Irbaz Bin Riaz ${ }^{5}$, and Faiz Anwer ${ }^{*}, 1$ \\ ${ }^{1}$ Department of Medicine, Hematology and Oncology, University of Arizona, Tucson, Arizona \\ ${ }^{2}$ Graduate student, College of Public Health, University of Arizona, Tucson, Arizona \\ ${ }^{3}$ College of Pharmacy, Arizona Cancer center, University of Arizona, Tucson, AZ, 85721 \\ ${ }^{4}$ Center for Health Outcomes \& Pharmaco-Economic Research, UA College of Pharmacy UA \\ College of Pharmacy \\ ${ }^{5}$ Mayo Clinic, Department of Hematology and Oncology, Rochester, Minnesota
}

\subsection{Introduction}

\begin{abstract}
Multiple Myeloma (MM) is characterized by monoclonal proliferation of plasma cells in the bone marrow. Based on cytogenetics, disease can be classified as high risk: $t(14 ; 16)$, $\mathrm{t}(14 ; 20)$, and/or del 17p13), intermediate risk: $\mathrm{t}(4 ; 14)$ and/or (1q) gain, and standard risk: trisomies, $\mathrm{t}(11 ; 14)$ and/or $\mathrm{t}(6 ; 14)$.[1] Consensus based treatment guidelines recommend 4 cycles of induction therapy such as bortezomib, lenalidomide and dexamethasone (VRd) followed by autologous stem cell transplantation therapy (ASCT) in transplant eligible patients with newly diagnosed multiple myeloma (NDMM). ${ }^{[1]}$ Some guidelines such as mSMART recommend patients with high risk NDMM should be given carfilzomib based triple combination induction regimen. In patients with second or higher relapses, treatment
\end{abstract}

\footnotetext{
*Corresponding Author Faiz Anwer MD, FACP, Director, Adult Blood \& Marrow Transplantation, Department of Medicine, Division of Hematology, Oncology. University of Arizona, Tucson, AZ, 85724, Phone: 520-626-3191, Fax: 520-626-8944, anwerf@email.arizona.edu.

$a_{\text {Equal contribution as first co-authors. }}$

AM (co-first author): literature search, figures, study design, data collection, data analysis, data interpretation, writing, editing VK (co-first author): literature search, figures, study design, data collection, data analysis, data interpretation, writing, editing AL (co-first author): literature search, figures, study design, data collection, data analysis, data interpretation, writing, editing AI: literature search, figures, study design, data collection, data analysis, data interpretation

UZ: data interpretation, writing, editing

$\mathrm{AM}^{\Delta}$ : data interpretation, writing, editing

IA: data interpretation, writing, editing

IBR: data interpretation, writing, editing:

FA (corresponding author): literature search, study design, data analysis, data interpretation, writing, editing
}

Conflict of interest statement Authors declare that there is no conflict of interest with this manuscript. The authors have no relevant affiliations or financial involvement with any organization or entity with a financial interest in or financial conflict with the subject matter or materials discussed in the manuscript. This includes employment, consultancies, honoraria, stock ownership or options, expert testimony, grants or patents received or pending, or royalties. No writing assistance was utilized in the production of this manuscript. For full disclosure, Dr. McBride discloses financial interest from Seattle Genetics in the form of advisory board membership. Dr. Anwer discloses financial interest from Seattle Genetics and Incyte Corporation in the form of advisory board membership and participation in speakers' program.

All other authors have nothing to disclose. 
depends on prior therapy, comorbidities (peripheral neuropathy, renal failure), marrow functioning as indicated by blood counts, and rapidity of relapse. Combination therapy incorporating proteasome inhibitors, immunomodulators, steroids and alkylating agents is generally recommended. ${ }^{[1]}$

Over the past two decades, advancements in MM therapy have markedly improved disease outcome and overall survival (OS). According to national institute of health (NIH) cancer statistics death rates due to MM have declined on average $0.7 \%$ each year over 2005-2014.

${ }^{[2]}$ Despite noticeable improvement in disease outcome, MM remains incurable with high rates of relapses, highlighting the unmet need for new treatment strategies. Proteasome inhibitor bortezomib was approved by the US Food and Drug Administration (FDA) for treatment of multiple myeloma in 2003. ${ }^{[3]}$ Despite its notable efficacy there are serious issues of side effects such as peripheral neuropathy (PNP), which has been reported in up to $30 \%$ of patients treated with bortezomib based regimens. ${ }^{[4,5]}$ Risk of PNP with bortezomib has been mitigated with subcutaneous and once weekly administration. ${ }^{[6]}$ In 2012, carfilzomib (CFZ), a novel proteasome inhibitor, was approved for treatment of relapsed and refractory multiple myeloma (RRMM) ${ }^{[7]} \mathrm{CFZ}$ is highly selective, irreversible epoxy-ketone molecule that targets chymotrypsin like activity of $20 \mathrm{~S}$ proteasome leading to cellular apoptosis which is particularly beneficial in malignant cells. CFZ has minimal off target activity causing fewer side effects including lower rates of PNP. ${ }^{[5,8]}$ Further, CFZ has demonstrated activity in bortezomib resistant cell lines. ${ }^{[7,9,10]}$. Since 2012 , CFZ has been studied in various clinical trials. The aim of our study is to conduct comprehensive literature search for efficacy, dosing and toxicity profile of CFZ in both newly diagnosed and relapsed setting. Our secondary aim is to analyze whether CFZ treatment can be extended to the frontline setting.

\subsection{Methods}

\subsection{Literature search}

A comprehensive literature search was performed on 6/5/2017 in the following resources: PubMed, EMBASE, Wiley Cochrane library, Scopus, Web of Science, CINAHL, and Clinicaltrials.gov. Search results were not limited to any geographical area or language, in the case English translations were available. However, studies done only after 2007 were included. Example search strategy is provided in appendix 1. Relevant articles from following conference proceedings were also included: the European Hematology Association, the American Society of Hematology, the American Society of Oncology and the American Society of Bone Marrow Transplantation.

\subsection{Eligibility criteria}

Studies fulfilling the following criteria were included: (1) Phase II or III clinical trials (2) Clinical trials from last 10 years (Jan 2007 till June 2017) (3) Studies that have efficacy outcomes clearly documented and (4) Studies focusing on CFZ as primary drug therapy. 


\subsection{Study Selection}

Relevant studies were reviewed by three independent reviewers (A. M., A. L., V. K.) based on title and abstract. Potentially relevant articles were screened through full text by aforementioned reviewers. Any conflicts were resolved with discussion.

\subsection{Data extraction and analysis}

Data was extracted on pre-specified tables which included following parameters: Author, year, study design, number of patients, median age, MM staging and cytogenetics, CFZ regimen, dose, median number of cycles, and efficacy outcomes (complete response [CR], near complete response [nCR], stringent complete response [sCR], very good partial response [VGPR], partial response [PR], overall response rate [ORR], overall survival [OS] and progression free survival [PFS]). If desired data was not reported in study, we documented it as not specified (NS).. Data was recorded as median or percentage.

\subsection{Results}

\subsection{Search results}

The literature search identified a total of 1835 articles. Additional 4 articles were identified through citation analysis. After excluding 630 duplicate studies, remaining 1209 were screened for relevance based on titles and abstracts. 89 studies were found potentially useful for our study after excluding 1120 studies. After reading full texts of remaining articles, further 63 articles were excluded due to one or more of the following reasons: phase 1 study, interim analysis, not focused on CFZ, full text not available, duplicate study, outcome not measured or not a clinical trial. 26 articles met the inclusion criteria, 15 in NDMM group and 11 in RRMM group. Summary of this selection process is given in PRISMA flow chart (Figure 1).

\subsection{Study demographics}

26 articles included a total of 5980 patients: 4205 in RRMM group and 1775 in NDMM group.

\subsection{Group A: Carfilzomib based regimens in newly diagnosed multiple myeloma (Table 1)}

\subsection{Carfilzomib based triplet regimens}

4.1.1. Carfilzomib, lenalidomide and dexamethasone (CRd) ( 5 studies, $n=268$ )

-We summarized five studies [11] [12] [13] [14, 15] with total of 268 NDMM patients who were treated with CRd. Roussel et al. (2016) ${ }^{[11]}$ conducted a phase II study $(\mathrm{n}=46)$ to assess efficacy of CRd regimen. All patients underwent 4 cycles of CRd induction, 43 patients received ASCT, 41 patients received further 4 cycles of consolidation therapy followed by 1 year of Len maintenance therapy in 27 patients. The dose of CFZ was $20 \mathrm{mg} / \mathrm{m}^{2}$ on D1, D2 of cycle 1 followed by $36 \mathrm{mg} / \mathrm{m}^{2}$ in subsequent cycles. There was no grade $\geq 3$ PNP. Cardiovascular AE were reported in 20 patients including 7 cardiac and 13 thrombotic events. $^{[11]}$ 
Zimmerman et al. (2016) ${ }^{[12]}$ in their phase I/II trial studied CRd regimen in 75 NDMM patients. 72 patients received 4 cycles of CRd induction followed by ASCT in 71 patients, 4 cycles of CRd consolidation in 66 patients and 10 additional cycles of CRd maintenance in 44 patients. Response was evaluated in 73 patients. VGPR and CR after consolidation were $96 \%$ and $73 \%$ respectively. After median follow-up of 17.5 months, PFS and OS of 2 years were $97 \%$ and $99 \%$ respectively. Peripheral neuropathy of only grade 1 or 2 was observed in $39 \%$ of patients. Most commonly reported grade $\geq 3$ adverse events (AE) were lymphopenia (28\%), neutropenia (18\%) and infections $(8 \%){ }^{[12]}$

Korde et al. (2013). ${ }^{[13]}$ conducted a phase II trial on 41 NDMM patients using CRD. Four cycles of induction with CRd were followed by ASCT in eligible patients. After 4 cycles of consolidation therapy, patients received 24 cycles of Len maintenance. Efficacy and toxicity data was evaluated in 38 patients. 25 out of 38 patients completed 8 cycles of CRd. No incidence of peripheral neuropathy was reported. Cardiovascular events of grade $\geq 3$ were reported in $8 \%$ of patient population. ${ }^{[13]}$

Jasielec et al. (2013) ${ }^{[14]}$ conducted a phase II study in 53 NDMM patients with CRd regimen. 53 patients received a median of 24 cycles followed by Len maintenance for a median of 12 months. After 24 cycles of therapy CR and sCR were 64\% and 55\% respectively. After follow-up of 31 months 3-year PFS and OS were 76\% and 96\% respectively. Toxicity data was not available. Estimated PFS and OS in patients with high risk cytogenetics were lower than in patients with standard risk cytogenetics $69 \%$ vs $88 \%$, and $83 \%$ vs $100 \%$ respectively. ${ }^{[14]}$

Jakubowiak et al. (2012) ${ }^{[15]}$ conducted a phase I/II study in 53 NDMM patients with CRd regimen. Patients received 8 cycles of induction therapy followed by 16 cycles of CRd maintenance therapy $(n=36)$ and off protocol single agent Len therapy $(n=5)$. Observed nCR and VGPR after $12+$ cycles were $72 \%$ and $100 \%$ respectively. After follow-up of 13 months, 2 -year PFS was $92 \%$. There was no grade $\geq 3$ PNP. In 2014, they presented updated followup outcomes in a subgroup of 23 elderly patients with median age of 72 years. ${ }^{[16]}$ All patients were given 24 cycles of CRd with CFZ dose of $20 \mathrm{mg} / \mathrm{m}^{2}$ in 2 patients, $27 \mathrm{mg} / \mathrm{m}^{2}$ in 4 patients and $36 \mathrm{mg} / \mathrm{m}^{2}$ in 17 patients. Reported $\searrow$ minimal response was as follows: All patients achieved at least a PR (100\%) and 91\% achieved at least a VGPR. CR, nCR, and sCR were $79 \%, 87 \%$ and $65 \%$ respectively. After follow-up of 30 months estimated PFS and OS were $80 \%$ and $100 \%$ respectively.

5 studies on CFZ regimen have reported promising efficacy outcomes with VGPR ranging from $20-96 \%$, CR of 45 to $73 \%$, ORR of $96 \%$ to $98 \%$, OS of $83 \%$ to $97 \%$ and 2-year PFS of $92 \%$ to $97 \%$. Triple combination (CRd) maintenance was used in Jakubowiak and Zimmerman et al's study.

4.1.2. Carfilzomib, thalidomide and dexamethasone ( $K T d)$ : ( 3 studies, $n=260)$ We included three studies. ${ }^{[17]}[18,19](n=260)$ that tested KTd in frontline setting.

Sonneveld et al. (2012) ${ }^{[19]}$ conducted a phase II trial on 58 patients with KTD to evaluate efficacy of KTD as frontline treatment for NDMM patients who were transplant eligible. Patients received 4 cycles of induction with KTD followed by high dose melphalan 
$\left(200 \mathrm{mg} / \mathrm{m}^{2}\right)$ and ASCT. After SCT patients underwent 4 cycles of KTD consolidation. Patients were divided into two cohorts. Dose of CFZ was $20 \mathrm{mg} / \mathrm{m}^{2}$ on D1/D2 of 1 st cycle (in both cohorts) followed by $27 \mathrm{mg} / \mathrm{m}^{2}$ (1st cohort) and $36 \mathrm{mg} / \mathrm{m}^{2}$ (2nd cohort) in subsequent cycles. 37 patients completed KTD induction, 31 patients underwent SCT followed by consolidation in 17 patients. Peripheral neuropathy grade $\geq 2$ was observed in 7 (17\%) patients. No hematological toxicity was reported. ${ }^{[19]}$

Sonneveld et al. (in 2015). ${ }^{[17]}$ conducted a phase II trial ( $\left.\mathrm{n}=91\right)$ using KTd as induction and consolidation therapy. Patients were divided into four cohorts based on carfilzomib doses. During induction and consolidation therapy, $20 \mathrm{mg} / \mathrm{m}^{2} \mathrm{CFZ}$ was infused over 2-10 minutes on D1/D2 of cycle 1 (all 4 dosing cohorts) which was escalated to $27 \mathrm{mg} / \mathrm{m}^{2}$ (1st cohort), $36 \mathrm{mg} / \mathrm{m}^{2}$ (2nd cohort), $45 \mathrm{mg} / \mathrm{m}^{2}$ (3rd cohort) and $56 \mathrm{mg} / \mathrm{m}^{2}$ (4th cohort) in the following cycles. After KTd induction therapy eligible patients received melphalan $200 \mathrm{mg} / \mathrm{m}^{2}$ and underwent ASCT. Following ASCT patients received 4 cycles of KTd consolidation therapy. There was no grade $\geq 3$ PNP. Cardiac and hematologic AE of grade $\geq 3$ were observed in $5 \%$ of patients. ${ }^{[17]}$

Wester R et al. $(2016)^{[18]}(\mathrm{n}=111)$ conducted a phase II trial using KTd. 4 cycles of KTd induction therapy were initiated at CFZ dose of $20 \mathrm{mg} / \mathrm{m}^{2}$ on D1/D2 of 1st cycle in four cohorts followed by dose escalation to $27 \mathrm{mg} / \mathrm{m}^{2}, 36 \mathrm{mg} / \mathrm{m}^{2}, 45 \mathrm{mg} / \mathrm{m}^{2}$ in $2 \mathrm{nd}$, 3rd and 4th cohort respectively. After 4 cycles of induction, patients received high dose melphalan (HDM) and ASCT followed by 4 cycles of consolidation therapy. Results in general were comparable between all cohorts. Common AEs were non-hematological. Grade $\geq 3 \mathrm{AE}$ included respiratory disorders (15\%) and GIT disorders (13\%). Cardiac AEs were seen in $4 \%$ of patients. ${ }^{[18]}$

Results of aforementioned three clinical trials on KTd regimen for NDMM could be summarized to as follows: CR ranging from 35 to $64 \%$ after consolidation therapy, VGPR ranging from 70 to $89 \%$ after consolidation therapy, ORR ranging from 90 to $95 \%$ and 1year, 2-year and 3-year PFS of 97\%, 71\% and 72\% respectively.

\subsubsection{Carfilzomib, cyclophosphamide and low dose dexamethasone (CCd) (3 studies/n=116)—There were three studies ${ }^{[20-22]}$ on CCd including 116 NDMM patients.}

Palumbo et al. (2014) ${ }^{[20]}$ conducted a phase I/II study in 28 NDMM patients. During phase I portion, CFZ was given to twelve patients at escalating doses of $36 \mathrm{mg} / \mathrm{m}^{2}, 45 \mathrm{mg} / \mathrm{m}^{2}$ and 70 $\mathrm{mg} / \mathrm{m}^{2}$ once weekly for 1 cycle to determine maximum tolerated dose (MTD). During phase II, CFZ at $70 \mathrm{mg} / \mathrm{m} 2$ was given once weekly for 9 cycles followed by consolidation therapy with $70 \mathrm{mg} / \mathrm{m}^{2}$ of CFZ. Incidences of CR and VGPR after 4 cycles on induction therapy were 83 and $22 \%$ respectively. ORR was $87 \%$. Common AE of grade $\geq 3$ were hematologic that included neutropenia (12\%) and anemia (12\%). ${ }^{[20]}$

Bringhen et al. (2013) ${ }^{[22]}$, conducted a phase II study on 58 patients using CCd regimen. CFZ was used at dose of $20 \mathrm{mg} / \mathrm{m}^{2}$ on D1 /D2 of 1 st cycle and $36 \mathrm{mg} / \mathrm{m}^{2}$ in subsequent 9 cycles, followed by maintenance with CFZ until progression of disease or intolerance due to toxicity. Response was evaluated in 41 patients after at least 4 cycles and toxicity data was 
evaluated in 51 patients after at least 1 cycle. Commonly observed non-hematological toxicities of grade $\geq 3$ were infections (7\%), renal (4\%), cardiac (4\%), and gastrointestinal complications $(2 \%)$.

Bringhen S et al. (2015) ${ }^{[21]}$, conducted a phase I/II study in 30 patients using CCd regimen. CFZ at doses of $40 \mathrm{mg} / \mathrm{m}^{2}, 56 \mathrm{mg} / \mathrm{m}^{2}$ and $70 \mathrm{mg} / \mathrm{m}^{2}$ on days 1,8 and 15 was used in a $3+3$ dose escalation scheme in combination with cyclophosphamide and dexamethasone. In 28 response evaluable patients at the end of 9 cycles VGPR and PR were $92 \%$ and $100 \%$ respectively. Commonly observed grade $\geq 3$ AEs were neutropenia (10\%), acute pulmonary edema (8\%), fatigue (3\%) and nausea (3\%). No PNP was observed.

Data on CCd regimen for NDMM was insufficient and heterogenous. Incidences of CR and VGPR after consolidation therapy was reported $17,53 \%$ and 100, $20 \%$ respectively in 2 trials by Bringhen et al.

4.1.4. Carfilzomib, melphalan and prednisone (CMP)—An ongoing phase III trial (NCT01818752) ${ }^{[23]}$ by Harper et al. has compared CMP with VMP (bortezomib, melphalan and prednisone) in 955 transplant ineligible NDMM patients. After follow-up of 54 weeks median PFS was 22.3 months in CFZ group vs 22.1 months in bortezomib group. Incidence of PNP was $2.5 \%$ (CFZ group) vs 35\% (bortezomib group). Grade $\geq 2$ AEs were seen in $74.7 \%$ of patients in CFZ group vs $76.2 \%$ of patients in bortezomib group.

Moreau P et al. $(2015)^{[8]}$, conducted a phase I/II study with CMP regimen (CFZ, melphalan and prednisone) in 50 patients. Twenty-four patients were enrolled in phase I and were divided into 4 cohorts based on CFZ dose. IV CFZ was given at doses of $20 \mathrm{mg} / \mathrm{m}^{2}(1 \mathrm{st}$ cohort), $27 \mathrm{mg} / \mathrm{m}^{2}$ (2nd cohort), $36 \mathrm{mg} / \mathrm{m}^{2}$ (3rd cohort), and $45 \mathrm{mg} / \mathrm{m}^{2}$ (4th cohort) for a 42 day cycle. During phase I MTD was defined as $36 \mathrm{mg} / \mathrm{m}^{2}$. Further 44 patients were enrolled in phase II portion of the study. The most common hematologic AEs were neutropenia $(38 \%)$, anemia (35\%) and thrombocytopenia (28\%).

\subsection{Carfilzomib based quadruplet regimens}

\subsubsection{Car-BiRD (carfilzomib, clarithromycin, lenalidomide and low dose} dexamethasone): $\left(\mathbf{2}\right.$ studies $/ \mathbf{n}=\mathbf{1 1 2}$ )-Forsberg et al. $(2016)^{[24]}$ conducted a phase II trial with Car-BiRd regimen in 72 patients. During induction phase, patients received CFZ and dex followed by ASCT. Following ASCT, 57 patients underwent consolidation with BiRD (Clarithromycin, Len and dex) regimen. After consolidation, 54 patients received 2year lenalidomide (Len) maintenance. CFZ was used at dose of $20 \mathrm{mg} / \mathrm{m}^{2}$ for 30 minutes during D1/D2 of 1st cycle followed by $45 \mathrm{mg} / \mathrm{m}^{2}$ which was escalated to $56 \mathrm{mg} / \mathrm{m}^{2}$ after enrolment of 1 st 26 patients until maximum response was achieved. Results were evaluated in 70 patients. ORR was achieved in $94 \%$ patients. The rate of $\mathrm{CR} / \mathrm{sCR}$ of $13 \%$ with $\mathrm{Kd}$ induction improved to $28 \%$ with BiRD consolidation and to $48 \%$ with Len maintenance. Most of the observed AEs were of grade 1 or 2 . PNP was seen in $31 \%$ of patients, all grade 1 or 2. Commonly observed grade $\geq 3$ toxicities were infections $(17 \%){ }^{[24]}$

Mark et al. (2014) ${ }^{[25]}$ conducted a phase II trial in 40 NDMM patients with Car-BiRd therapy. Patients received IV CFZ at dose of $20 \mathrm{mg} / \mathrm{m}^{2}$ for 30 minutes during D1/D2 of 1 st 
cycle and $45 \mathrm{mg} / \mathrm{m}^{2}$ in subsequent cycles. After enrolment of 26 patients, dose was escalated to $56 \mathrm{mg} / \mathrm{m}^{2}$. ASCT was performed in eligible patients and consolidation phase with BiRd was initiated. While transplant ineligible patients were started directly with BiRd therapy followed by Len maintenance. Results were evaluated in 36 patients. Grade $\geq 2$ AEs seen were cardiovascular (5\%) and renal (8\%). ${ }^{[25]}$ Treatment with Car-BiRD did not yield impressive results especially when compared to other treatment options available. Incidences of CR and VGPR after consolidation therapy were $0 \%, 28 \%$ of CR and $57 \%, 67 \%$ of VGPR in above mentioned two studies.

\subsubsection{Carfilzomib, cyclophosphamide, thalidomide and low dose} dexamethasone (CYKLONE)-Mikhael et al. (2015) ${ }^{[26]}$ conducted a phase Ib/II trial with CYKLONE (CFZ, cyclophosphamide, thalidomide and dex) regimen in 64 NDMM patients. Four cycles of induction therapy in all patients were followed by SCT in 34 patients. After SCT, patients received 8 cycles of consolidation. Dose of carfilzomib was 15$20 \mathrm{mg} / \mathrm{m}^{2}$ in 3 patients, $20-27 \mathrm{mg} / \mathrm{m}^{2}$ in 25 patients, $20-36 \mathrm{mg} / \mathrm{m}^{2}$ in 29 patients and $20-45$ $\mathrm{mg} / \mathrm{m}^{2}$ in 7 patients. ORR was $91 \%$. In this regimen, most grade 1 PNP (31\%) was said to be primarily related to thalidomide treatment. There was no grade $\geq 3$ PNP. Grade $\geq 1$ cardiopulmonary AEs (Cough (6\%) and dyspnea (20\%)) were observed in 5\% of patients. ${ }^{[26]}$ Quadruplet regimens didn't yield any greater benefit as compared to triplet regimen for treatment of NDMM. In fact, incidence of PR and VGPR were low with Car-BiRD and CYKLONE when compared to KRd or KTd regimens.

\subsection{Group B: Carfilzomib based regimens in relapsed and refractory multiple myeloma (Table 2)}

\subsection{Single agent carfilzomib ( 5 studies, $n=807$ )}

5.1.1. Phase III (1 study, $\mathbf{n}=\mathbf{3 1 5}$ )—Hajek et al. ${ }^{[27]}$ (2016) compared single agent carfilzomib with best supportive treatment (low dose corticosteroids and optional cyclophosphamide) in heavily pretreated RRMM patients with median 5 prior lines of therapy. A total of 315 patients were randomized into CFZ group ( $n=157)$ and control group $(\mathrm{n}=158)$. Primary end-point was OS (time form randomization to death from any cause) which was not statistically different in 2 groups (10.2 months vs 10 months in carfilzomib and control group respectively, HR 0.975). The most common grade $\geq 3 \mathrm{AE}$ were anemia (26 vs $31 \%$ ), neutropenia (8 vs $12 \%$ ) and thrombocytopenia ( 24 vs $22 \%$ ) with CFZ vs control group. Incidence of any grade hypertension (15 vs $6 \%$ ) and grade $\geq 3$ grouped renal failure events were higher in CFZ group than control group (24 vs 9\%). ${ }^{[27]}$

5.1.2. Phase II studies (4 studies, $\mathbf{n}=491$ ) —Jagannath et al. ${ }^{[10]}$ (2012) performed the first phase II trial on single agent CFZ in 46 RRMM patients who had received median of 5 prior lines of therapy. Median duration of response (i.e. from time of $\geq \mathrm{PR}$ to confirmed disease progression or death from any cause) was 7.2 months. Significant grade $3 / 4$ adverse events were anemia (37\%), lymphopenia (28.3\%), thrombocytopenia (26\%), renal failure $(13 \%)$ and pneumonia (11\%).

Siegel et al. ${ }^{[7]}$ (2012) used $20-27 \mathrm{mg} / \mathrm{m}^{2}$ dose of CFZ to study its efficacy in heavily pretreated 266 multiple myeloma patients. Patients had median 5 prior lines of therapy 
including bortezomib, thalidomide and lenalidomide. Most common toxicities were hematologic (anemia 46\% and thrombocytopenia 39\%). Incidence of all grade peripheral neuropathy was $12 \%$. CR achieved was $0.4 \%$.

Watanabe et al. ${ }^{[28]}$ (2016) performed phase II study on single agent CFZ with $15 / 20$ and $20 / 27 \mathrm{mg} / \mathrm{m}^{2}$ dose in 50 patients. Patients had median 5 prior lines of therapy. ORR in patients who received $20 / 27 \mathrm{mg} / \mathrm{m}^{2} \mathrm{CFZ}$ was $22.5 \%$ comparable to $23.7 \%$ in Siegel et al.'s study.[7] 88\% experienced at least one grade $\geq 3 \mathrm{AE}$, the most common of which were lymphopenia (68\%), neutropenia (38\%), anemia (30\%) and $26 \%$ of each thrombocytopenia and leucopenia. Incidence of PN was $16 \%$ all less than grade 3. 50\% of those who developed $\mathrm{PN}$, already had PN at baseline. They reported higher incidence of hypertension (HTN) ( $20 \%$ vs $14.3 \%$ in Siegel et al.'s study) with $8 \%$ experiencing grade $\geq 3$ HTN. $16 \%$ discontinued treatment with infection being the most common cause for it. Incidence of grade $\geq 3$ cardiac AE was $2 \%$ as compared to $9.5 \%$ in Siegel et al.'s study. ${ }^{[7]}$

Vij et al. ${ }^{[9]}$ (2012) studied efficacy of single agent CFZ in bortezomib naïve patients with less heavily treated MM (1 to 3 prior lines of therapy). They divided total cohort of 129 bortezomib naïve patients in 2 groups. Cohort 1 received $20 \mathrm{mg} / \mathrm{m}^{2} \mathrm{CFZ}$ for all cycles of treatment. Cohort 2 received $20 \mathrm{mg} / \mathrm{m}^{2}$ for cycle 1 and $27 \mathrm{mg} / \mathrm{m}^{2}$ for remaining cycles of treatment. Overall incidence of PN was $17 \%$ with only one patient experiencing grade $3 \mathrm{PN}$.

With single agent CFZ in patients with median 5 prior lines of therapy, efficacy outcomes were reported as follows: CR of $0 \%$ to $1 \%$, VGPR of 3 to $5.1 \%$, ORR of $16.7 \%$ to $23.7 \%$ and median PFS ranging from 3.5 to 5.1 months.

\subsection{Carfilzomib based doublet regimens}

\subsubsection{Phase II studies ( 3 studies, $n=204$ )}

Carfilzomib + panobinostat: Berdeja et al. ${ }^{[29]}$ (2015) performed phase I/II study on combination of CFZ and panobinostat in 44 RRMM patients who had median 5 prior lines of therapy. Patients were treated at maximum tolerable dose of $30 \mathrm{mg}$ panobinostat and $20 / 45 \mathrm{mg} / \mathrm{m}^{2}$ of CFZ until disease progression or intolerable toxicity. $59 \%$ patients had toxicity related dose reductions for panobinostat. Out of 44 patients, 35 patients discontinued treatment ( $41 \%$ due to disease progression, $20 \%$ due to patient's/physician's decision, $9 \%$ due to treatment related side effects mainly cardiotoxicity and $9 \%$ due to nontreatment related side effects). One patient died due to progressive disease and one due to heart failure.

Carfilzomib + dexamethasone: Berenson et al. $(2014)^{[30]}$ performed phase I/II study on 116 RRMM patients to investigate efficacy of $70 \mathrm{mg} / \mathrm{m}^{2}$ once weekly CFZ plus dexamethasone. Berenson et al. evaluated 30-min infusion to investigate whether longer infusion time allows administration of higher doses. ORR was 77\% and median PFS 12.6 months. Most common adverse effects of any grade were hematologic (anemia 28\%, thrombocytopenia $22 \%$ and neutropenia $10 \%$ ). Common grade $\geq 3 \mathrm{AE}$ were fatigue $11 \%$, HTN 7\%, pneumonia $6 \%$ and acute kidney injury $6 \%$. Rates of grade $\geq 3$ dyspnea, cardiac failure, and peripheral neuropathy were $5 \%, 2 \%$, and $1 \%$, respectively. ${ }^{[30]}$ 
Lendvai et al. ${ }^{[31]}$ (2014) studied efficacy and AE profile of 20/56mg/m² dose of CFZ in 44 heavily pretreated RRMM patients who had median of 5 prior lines of therapy.. Incidences of CR, VGPR, PR, ORR, median OS and median PFS were 2\%, 21\%, 31\%, 55\%, 20.3 months and 4.1 months respectively. Most common grade 3/4 AE were hematologic (lymphopenia 43\%, thrombocytopenia 32\% and 18\% for each of anemia, leukopenia and neutropenia). Most common grade 3/4 non-hematologic AE were HTN (25\%), pneumonia $(18 \%)$ and heart failure (20\%). Out of $25 \%(\mathrm{n}=11)$ who developed grade $3 / 4 \mathrm{HTN}$, one patient developed nephrotic range proteinuria, another developed thrombotic microangiopathy and 4 developed heart failure. Incidence of PNP was 16\%, all grade 1. AEs related discontinuations were most commonly due to decline in left ventricular systolic function (5 patients). 39\% patients required dose reductions with HTN being the most common cause.

With CFZ based doublet regimens in patients with 5 prior lines of therapy, efficacy outcomes were as follows: 2 VGPR ranging from 23 to $33 \%$, ORR ranging from 55 to $67 \%$ and median PFS of 4.1 months and 7.7 months (at median follow up of 17 months).

\subsubsection{Phase III (1 study, $n=929)$}

ENDEAVOR [carfilzomib plus dexamethasone $(\mathrm{Kd})$ vs bortezomib plus dexamethasone (Vd)]: ENDEAVOR study by Dimopoulos et al. (2016) ${ }^{[32]}$ is the largest (929 patients) multi-center phase 3 trial on RRMM. They did head to head trial on CFZ plus dexamethasone versus bortezomib plus dexamethasone. Results of preplanned interim analysis have been reported. 929 patients were randomized into 2 groups: 464 in CFZ and 465 in bortezomib group. Patients had received median of 2 prior regimens. CFZ was given at doses of 20 and $56 \mathrm{mg} / \mathrm{m}^{2}$ infused over 30-min. In the bortezomib group, 79\% received it subcutaneously while $21 \%$ intravenously. Primary endpoint was PFS which was double (18.7 months) with CFZ compared to bortezomib (9.4 months). The most common $\geq$ grade 3 $\mathrm{AE}$ in CFZ and bortezomib group respectively were anemia (14\% vs $10 \%$ ), HTN (9\% vs $3 \%$ ), thrombocytopenia ( $8 \%$ vs $9 \%$ ) and pneumonia (7\% vs $8 \%$ ). The percentage of grade $\geq$ $2 \mathrm{PN}$ was significantly higher in bortezomib group (32\%) than CFZ group (6\%). Serious adverse events were more frequently reported in CFZ group (48\%) than bortezomib group (36\%). Incidence of many AE (noticeably dyspnea, cough, grade 3/4 HTN (9\% vs 3\%), pyrexia, any grade cardiac failure, any grade renal failure and muscle spasms) was higher with CFZ.

\section{ENDEAVOR subgroup analyses}

Impact of prior treatment: Moreau and colleagues ${ }^{[33]}$ performed subgroup analysis of ENDEAVOR to study effect of prior treatment and number of prior lines of therapy. Median PFS for Kd vs Vd in patients who had one prior therapy was 22.2 vs 10.1 months respectively; with $\geq 2$ prior lines of therapy, 14.9 vs 8.4 months respectively; with prior bortezomib exposure, 15.6 vs 8.1 months respectively and with prior lenalidomide exposure, 12.9 vs 7.3 months respectively.

Impact of cytogenetic risk: Chang et al. ${ }^{[34]}$ performed subgroup analysis of ENDEAVOR to evaluate whether cytogenetic risk had an impact on outcome with Kd vs Vd. Median PFS 
was 8.8 months vs 6.0 months for $\mathrm{Kd}$ vs $\mathrm{Vd}$ in the high-risk group. In the standard-risk group median PFS was not estimable for Kd vs 10.2 months for Vd.

Impact of age: Ludwig and colleagues ${ }^{[35]}$ did subgroup analysis of ENDEAVOR to study efficacy outcomes in patients who had been grouped by age and received either Kd or Vd. PFS was superior with $\mathrm{Kd}$ than $\mathrm{Vd}$ in each subgroup ( $<65$ years: not estimable for $\mathrm{Kd}$ vs 9.5 months for Vd, 65-74 years: 15.6 months for Kd vs 9.5 months for $\mathrm{Vd}, \geq 75$ years: 18.7 months for Kd vs 8.9 months for $\mathrm{Vd}$ ). Interestingly, the eldest-age subgroup ( $\geq 75$ years) showed improved survival than two younger-age subgroups.

ENDEAVOR is the only available trial with head to head comparison of CFZ vs bortezomib, reported CR of $11 \%$ vs $4 \%$, VGPR of $42 \%$ vs $22 \%$, ORR of $77 \%$ vs $63 \%$, and median PFS of 18.7 months vs 9.4 months with Kd vs Vd respectively. Subgroup analyses of ENDEAVOR showed that improved survival was irrespective of age, prior transplant status, prior treatment, number of prior lines of therapy or cytogenetics. Inferior outcomes in patients with high risk cytogenetics suggest that poor prognostic outcome of unfavorable cytogenetics couldn't be overcome by either proteasome inhibitor.

\subsection{Carfilzomib based triplet regimens ( 2 studies, $n=448$ )}

\subsubsection{Phase II studies ( 1 study, $n=52$ )}

Carfilzomib, lenalidomide and low dose dexamethasone (CRd): Wang et al. ${ }^{[5]}$ (2013) did phase I/II study on 52 patients who were given $20-27 \mathrm{mg} / \mathrm{m}^{2} \mathrm{CFZ}$ for median of 9.5 cycles. Most common grade 3/4 adverse events were hematologic (lymphopenia 48\%, neutropenia $33 \%$, thrombocytopenia $19 \%$ and anemia $19 \%$ ). Incidence of overall cardiac adverse events was 19\% (16 patients) with 6 patients (7\%) experiencing $\geq$ grade 3 cardiac AE.

\subsubsection{Phase III studies ( 1 study, $n=396$ )}

\section{Carfilzomib + lenalidomide + dexamethasone (KRd) vs lenalidomide and}

dexamethasone (Rd): Stewart et al. ${ }^{[36]}$ (2014) compared safety and efficacy of KRd vs Rd in 792 RRMM patients randomized into two groups in 1:1 ratio. The dose of CFZ was $20 \mathrm{mg} / \mathrm{m}^{2}$ (initial dose) escalated to $27 \mathrm{mg} / \mathrm{m}^{2}$ (target dose) infused over 10 minutes. The trial has reached its primary endpoint at interim analysis. Primary endpoint was PFS which was 26.3 months for KRd vs 17.6 months for Rd. Any grade AE more frequently encountered with KRd group were HTN (14.3 vs 6.9 in Rd), hypokalemia (27.6 vs 13.4), muscle spasms, infection, nasopharyngitis, cough, upper respiratory tract infection, diarrhea and pyrexia. However, rates of discontinuations with these $\mathrm{AE}$ were very low $(<1 \%)$. Grade $\geq 3 \mathrm{AE}$ were HTN (4.3\% for CFZ vs $1.8 \%$ for control group), dyspnea (2.8\% vs $1.8 \%$ ) and cardiac failure (3.8\% vs $1.8 \%$ ). Rate of discontinuation was $69.9 \%$ and $77.9 \%$ for $\mathrm{KRd}$ vs $\mathrm{Rd}$ respectively. Most of them were due to disease progression (39.8\% and 50.1\%) or AE (15.3\% and $17.7 \%)$. Triple combination therapy (CRd) yielded highest efficacy among the trials reported so far for RRMM with CR of $17.7 \%$, VGPR of 70\%, ORR of $87 \%$ and median PFS of 26.3 months. CRd regimen in trial by Wang et al. resulted in outcomes less impressive than Stewart et al.'s results with CR of $1.9 \%$, VGPR of $36.5 \%$, ORR of $77 \%$ and median PFS of 15.4 months. Another randomized phase III trial by Moreau and colleagues ${ }^{[37]}$ comparing $\mathrm{KRd}$ with $\mathrm{Rd}$ is ongoing. 


\subsection{Toxicity profile of carfilzomib}

Incidence of grade $\geq 3 \mathrm{AE}$ was as follows: anemia(11-41\%), lymphopenia (5.7-50\%), thrombocytopenia (4-53\%), neutropenia (11-53\%), leukopenia (1.4-43\%), dyspnea (3.4$11 \%)$, pyrexia (1.5-2.4\%), URTI (1.8-5.7\%), increased creatinine (2.6-2.9\%), PN (1-1.1\%), hypophosphatemia $(1.7-16 \%)$, hyponatremia $(7.1-11 \%)$, pneumonia $(8.6-18 \%)$, renal failure (3.4-9\%), febrile neutropenia ( $0.8-7 \%)$, hypertension (3-25\%), heart failure (3.4$20 \%$ ) and hypokalemia (1.7 to 9.4\%).[38-44] Most common grade $\geq 3 \mathrm{AE}$ were hematologic. ENDEAVOR reported significantly higher incidence of $\geq 2$ PNP in bortezomib group (32\%) vs $6 \%$ in carfilzomib group. ENDEAVOR trial using serial echocardiograms reported no increased risk of cardiotoxicity and ventricular dysfunction with CFZ vs bortezomib.

\subsection{Discussion}

CFZ based therapies have been evaluated in RRMM setting, a population that is more difficult to treat than NDMM patients for multiple reasons. Based on literature summarized in this review, it is suggested that CFZ based treatment was very successful in the frontline setting. PN is a crippling condition that can develop as complication of disease itself or its treatment including agents like bortezomib and thalidomide. ${ }^{[45]}$ Reported incidence of bortezomib induced grade $\geq 3$ PNP $(2-23 \%)^{[46]}$ can impair life quality and the best way to preserve quality of life is to prevent development of peripheral neuropathy at the first place without any compromise on the efficacy of the regimen. A phase III trial ${ }^{[23]}$ (ongoing) with head to head comparison of CFZ + MP vs bortezomib + MP, reported no significant difference in PFS with CFZ vs bortezomib. However, observed incidence of PNP was $35.1 \%$ with bortezomib vs $2.5 \%$ with CFZ.

With ORR approaching higher than $95 \%$, CR rate close to $70 \%$, 2-year PFS $\geq 90 \%$ and 3year PFS $\geq 70 \%$, outcomes reported with CFZ are comparable if not higher to bortezomib based regimens but with much lower incidence of PNP.[23, 44, 47] This supports use of CFZ in frontline setting across the board as well as for high risk disease. From comparison of studies included in our systematic review, we reported best responses with CRd combination therapy with VGPR ranging from $20 \%-96 \%$, CR of $45 \%$ to $73 \%$, ORR of $96 \%$ to $98 \%$, OS of $83 \%$ to $97 \%$ and 2-year PFS of $92 \%$ to $97 \%$. Short term follow-up results from a phase I trial of four drug combination using daratumumab + KRd in 22 patients with NDMM produced very promising results with ORR of $100 \%$ (5\% CR and 86\% VGPR) and $100 \%$ six month PFS.[48] The idea of quadruplet regimen incorporating monoclonal antibody needs further exploration based on large scale trials with longer follow up. It should be kept in mind that due to population heterogeneity and differences in CFZ dosing, schedule and combination, comparison results can be imprecise. It is important to recognize, many studies in the CFZ group relied on frontline induction and melphalan high dose consolidation (consolidation 1) with autologous stem cell rescue followed by variable cycles (4 and higher) of CFZ with conventional dose consolidation (consolidation 2) intent and then patients were kept on maintenance for a variable duration. Triple combination (CRd) as a maintenance strategy was used in Jakubowiak and Zimmerman et al's study, this approach is not standard with limited available data on efficacy and safety. Idea of second conventional 
dose consolidation, either single agent CFZ or combination (CRd based) maintenance therapy needs further exploration in large randomized trials. Despite heavy use and exposure of CFZ, most studies had negligible incidence of grade > 3 PNP if at all present. Based on the limited data from two studies where head to head comparison was done, results suggest that CFZ demonstrates higher or equal efficacy to bortezomib with much favorable $\mathrm{AE}$ profile.[23, 44]

Car-BiRD regimen has not produced promising results in treatment of NDMM. It could be due to higher percentage (62\%) of their patients with high risk cytogenetics and lack of additional cycles of consolidation beyond use of high dose melphalan. In the presence of other options available especially KRd, Car-BiRD doesn't seem an attractive option for treatment of MM patients. Quadruplet regimens didn't yield ang greater benefit as compared to triplet regimen for treatment of NDMM. In fact, percentage of patients with PR and VGPR was less with Car-BiRD and CYKLONE when compared to KRd or KTd regimens. Daratumumab + KRd combination generated exceptionally good results with ORR of $100 \%$ in phase 1 setting and warrants further testing in larger randomized trials.

Carfilzomib is approved for treatment of RRMM in patients with 1-3 prior lines of therapy, either alone or in combination with lenalidomide \pm dexamethasone. Clonal heterogeneity is common in MM. Combination therapy helps decrease tumor burden despite clonal heterogeneity. CFZ based triplet combination therapy produced better outcomes than doublet regimen which in turn had better results than single agent CFZ. Phase III study by Hajek et al. didn't reach primary endpoint i.e. single agent CFZ has superior OS than supportive treatment in heavily pretreated MM.[27] Though single agent CFZ failed to significantly improve outcomes in these heavily pretreated patients, CFZ remains an active treatment option as combination therapy and in a variety of settings as suggested by ENDEAVOR, ASPIRE and other trials on CFZ in NDMM setting.

Higher incidence of hypertension with CFZ based therapy reported in some studies [27, 28, $32,49,50]$ is note-worthy. Proteasome inhibitors are postulated to cause cardiomyocyte apoptosis through accumulation of pre-apoptotic bodies.

MM patients treated with PI should have their blood pressure (BP) carefully monitored and controlled to alleviate risk of cardiotoxicity. Standard FDA approved dose of CFZ is $20 / 27 \mathrm{mg} / \mathrm{m}^{2}$ infused over 10 minutes. Higher dose $20 / 56 \mathrm{mg} / \mathrm{m} 2$ used by Lendvai et al. produced deeper and durable responses but with higher incidence of grade $3 / 4$ HTN. Berenson et al (2014) ${ }^{[30]}$ studied $70 \mathrm{mg} / \mathrm{m}^{2}$ per weekly dose of CFZ while Dimopoulos et al. ${ }^{[44]}$ used $56 \mathrm{mg} / \mathrm{m}^{2}$ twice weekly dose. Although cross trial comparisons can be imprecise due to differences in patient population (e.g. median no. of prior lines of therapy was 1 for Berenson's study vs 2 for ENDEAVOR study), ORR reported in 2 studies were comparable (77\% for both). However, median PFS was higher in ENDEAVOR study (18.7 vs 12.6 in Berenson study). An ongoing ARROW trial[51] is comparing $70 \mathrm{mg} / \mathrm{m}^{2}$ once weekly dose of CFZ to twice weekly $20 / 27 \mathrm{mg} / \mathrm{m}^{2}$ dose. Results of ARROW and further large-scale trials are needed to study benefit-to-risk profile of $20 / 56$ and $20 / 70 \mathrm{mg} / \mathrm{m}^{2}$ dosing of CFZ vs standard $20 / 27 \mathrm{mg} / \mathrm{m}^{2}$ dose. 


\subsection{Conclusion}

Our results suggest that CFZ demonstrates comparable efficacy to bortezomib with much favorable AE profile both in NDMM and RRMM. There are only two studies with head to head comparison of CFZ based regimens with bortezomib based regimens.[23, 44] Crosstrial comparisons of studies on CFZ with studies on bortezomib can be imprecise due to significant heterogeneity in patient population, number of prior lines of therapy, dose and schedule of drug used and whether treatment was in conjunction with stem cell transplantation.. KRd and Rd regimen have well documented efficacy for treatment of RRMM. Further large-scale trials are needed to study benefit-to-risk profile of $20-56$ and $20-70 \mathrm{mg} / \mathrm{m}^{2}$ dose of CFZ vs standard $20-27 \mathrm{mg} / \mathrm{m}^{2}$ dose. Reported incidence (3\%-25\%) of grade $\geq 3 \mathrm{HTN}$ with CFZ deserves attention and emphasizes the importance of serial BP monitoring before, during and after CFZ infusions. For patients with NDMM, data supporting KRd mainly comes from phase II trials. Deep, rapid and sustainable response using KRd with safer toxicity profile supports extension of KRd therapy to frontline therapy for all risk categories of MM. Role of conventional dose second consolidation after HDCT and autologous stem rescue needs further exploration for safety and efficacy in larger randomized trials. Data from randomized phase III trials is needed for head to head comparison of KRd vs RVd, and KRd vs daratumumab-KRd for NDMM patients.

\section{Acknowledgments}

Financial disclosure statement: This work was supported by grant P30 CA023074 (FA) from the National Cancer Institute, National Institutes of Health, Bethesda, MD.

We acknowledge the efforts of all investigators and their teams who worked on the original trials. We sincerely appreciate all myeloma patients and their families, who volunteered and participated in these clinical trials which are mentioned in our manuscript.

\section{Appendix 1}

Example Search Strategy ("carfilzomib"[Supplementary Concept] OR "carfilzomib"[All Fields]) AND ("multiple myeloma”[MeSH Terms] OR ("multiple”[All Fields] AND "myeloma"[All Fields]) OR "multiple myeloma"[All Fields]) AND ((Clinical Trial[ptyp] OR Meta-Analysis[ptyp] OR systematic[sb]) AND “2007/06/28”[PDat] : "2017/06/24” [PDat])

\section{Biographies}

AM is a medical doctor, who graduated from Rawalpindi Medical College (RMC), Pakistan. Dr. Adeela is working as research volunteer in Hematology/Oncology at University of Arizona Cancer Center (UACC).

AL has her MD and is a graduate from RMC, Pakistan. She is currently working as a research volunteer in Hematology/Oncology at UACC.

VK has his MD and is a graduate from RMC, Pakistan. He is working as a research volunteer in Hematology/Oncology at UACC. 
AI has his MD degree from RMC, Pakistan. He is working as a research volunteer in Hematology/Oncology at UACC.

UZ has his MD degree from Punjab medical college (PMC), Pakistan. He is a graduate student of MPH Biostatistics at the Mel \& Enid Zuckerman College of Public Health, University of Arizona. He is also a research associate at the Department of Medicine, Hematology/Oncology division at UACC.

IBR: had his MD from Nishtar Medical College, currently working as a Hem/Onc trainee fellow at Mayo Clinic, Department of Hematology and Oncology, Rochester, Minnesota.

AM has his PharmD and MS and is the Clinical Coordinator of Hematology/Oncology UACC.

IA is a chief scientist at Matrix45 and professor (part-time) at University of Arizona in the Department of Pharmacy Practice. He has lectured, consulted, and conducted research throughout the America, Europe, the Asia-Pacific region, and Africa.

FA has his MD, is team leader for Multiple Myeloma program, Clinical Director of the adult Blood and Marrow Transplant Program and the Director of the Stem Cell Harvest Program in the Division of Hematology/Oncology at UACC and is an Assistant Professor of Medicine at the University of Arizona, College of Medicine.

\section{References}

1. Strite S, et al. Comparing the efficacy of denosumab versus ZO-ledronic acid (ZA) for prevention of Skeletal-Related events (SRES): A critical appraisal of three pivotal trials. Supportive Care in Cancer. 2015; 23(1):S33.

2. Institute, N.C. Cancer Stat Facts: Myeloma.

3. Kane RC, et al. Velcade: U.S. FDA approval for the treatment of multiple myeloma progressing on prior therapy. Oncologist. 2003; 8(6):508-13. [PubMed: 14657528]

4. Mohty B, et al. Peripheral neuropathy and new treatments for multiple myeloma: Background and practical recommendations. Haematologica. 2010; 95(2):311-319. [PubMed: 20139393]

5. Wang M, et al. Final results from the phase Ib/II study (PX-171-006) of carfilzomib, lenalidomide, and low-dose dexamethasone $(\mathrm{CRd})$ in patients with relapsed or progressive multiple myeloma. Journal of Clinical Oncology. 2013; 31(15)

6. Rosinol L, et al. Superiority of bortezomib, thalidomide, and dexamethasone (VTD) as induction pretransplantation therapy in multiple myeloma: a randomized phase 3 PETHEMA/GEM study. Blood. 2012; 120(8):1589-96. [PubMed: 22791289]

7. Siegel DS, et al. A phase 2 study of single-agent carfilzomib (PX-171-003-A1) in patients with relapsed and refractory multiple myeloma. Blood. 2012; 120(14):2817-2825. [PubMed: 22833546]

8. Moreau P, et al. Phase 1/2 study of carfilzomib plus melphalan and prednisone in patients aged over 65 years with newly diagnosed multiple myeloma. Blood. 2015; 125(20):3100-3104. [PubMed: 25784682]

9. Vij R, et al. An open-label, single-arm, phase2 (PX-171-004) study of single-agent carfilzomib in bortezomib-naive patients with relapsed and/or refractory multiple myeloma. Blood. 2012; 119(24): 5661-5670. [PubMed: 22555973]

10. Jagannath S, et al. An open-label single-arm pilot phase II study (PX-171-003-A0) of low-dose, single-agent carfilzomib in patients with relapsed and refractory multiple myeloma. Clinical Lymphoma, Myeloma and Leukemia. 2012; 12(5):310-318. 
11. Roussel M, et al. Frontline therapy with carfilzomib, lenalidomide, and dexamethasone (KRd) induction followed by autologous stem cell transplantation, krd consolidation and lenalidomide maintenance in newly diagnosed multiple myeloma (NDMM) patients: primary results of the intergroupe francophone du myelome (IFM) krd phase II study. Blood. 2016; 128 Conference: 58th annual meeting of the american society of hematology, ASH 2016. United states. Conference start: 20161203. Conference end: 20161206.

12. Zimmerman T, et al. Final results of a phase 2 trial of extended treatment (TX) with Carfilzomib (CFZ), Lenalidomide (LEN), and Dexamethasone (KRd) plus autologous stem cell transplantation (ASCT) in newly diagnosed multiple myeloma (NDMM). Blood. 2016; 128(22)

13. Korde N, et al. Phase II clinical and correlative study of carfilzomib, lenalidomide, and dexamethasone followed by lenalidomide extended dosing (CRD-R) induces high rates of mrd negativity in newly diagnosed Multiple Myeloma (MM) patients. Blood. 2013; 122(21)

14. Jasielec $\mathrm{J}$, et al. Predictors of treatment outcome with the combination of carfilzomib, lenalidomide, and low-dose dexamethasone (CRD) in newly diagnosed multiple myeloma (NDMM). Blood. 2013; 122(21)

15. Jakubowiak AJ, et al. Carfilzomib, lenalidomide, low-dose dexamethasone (CRD) in elderly patients with newly diagnosed multiple myeloma (NDMM). Clinical Lymphoma, Myeloma and Leukemia. 2013; 13:S41-S42.

16. Dytfeld D, et al. Carfilzomib, lenalidomide, and low-dose dexamethasone in elderly patients with newly diagnosed multiple myeloma. Haematologica. 2014; 99(9):e162-e164. [PubMed: 24972772]

17. Sonneveld $\mathrm{P}$, et al. Phase 2 study of carfilzomib, thalidomide, and dexamethasone as induction/ consolidation therapy for newly diagnosed multiple myeloma. Blood. 2015; 125(3):449-456. [PubMed: 25398935]

18. Wester R, et al. Phase 2 study of carfilzomib, thalidomide, and low-dose dexamethasone as induction/consolidation in newly diagnosed, transplant eligible patients with multiple myeloma, the carthadex trial. Blood. 2016; 128(22)

19. Sonneveld P, et al. Carfilzomib combined with thalidomide and dexamethasone (CTD) is an highly effective induction and consolidation treatment in newly diagnosed patients with multiple myeloma (MM) who are transplant candidate. Blood. 2012; 120(21)

20. Palumbo A, et al. Weekly carfilzomib, cyclophosphamide and dexamethasone (wCCd) in newly diagnosed multiple myeloma patients: A phase I-II study. Blood. 2014; 124(21)

21. Bringhen $\mathrm{S}$, et al. Weekly carfilzomib in combination with cyclophosphamide and dexamethasone (wccyd) in patients (pts) with newly diagnosed multiple myeloma (NDMM): A phase 1/2 study of the european myeloma network trialist group. Clinical Lymphoma, Myeloma and Leukemia. 2015; 15:e70.

22. Bringhen S, et al. A phase II study with carfilzomib, cyclophosphamide and dexamethasone (CCd) for newly diagnosed multiple myeloma. Blood. 2013; 122(21)

23. ClinicalTrials.gov. Phase 3 Study of Carfilzomib, Melphalan, Prednisone vs Bortezomib, Melphalan, Prednisone in Newly Diagnosed Multiple Myeloma (CLARION). Jun.2017

24. Forsberg PA, et al. Carfilzomib induction with lenalidomide and clarithromycin consolidation and lenalidomide maintenance (CarBiRD) for multiple myeloma (MM). Blood. 2016; 128(22)

25. Mark TM, et al. Car-bird [carfilzomib, clarithromycin $\left(\right.$ biaxin $\left.^{\circledR}\right)$, lenalidomide/(revlimid $\left.{ }^{\circledR}\right)$, dexamethasone) for newly-diagnosed multiple myeloma. Blood. 2014; 124(21)

26. Mikhael JR, et al. Phase Ib/II trial of CYKLONE (cyclophosphamide, carfilzomib, thalidomide and dexamethasone) for newly diagnosed myeloma. Br J Haematol. 2015; 169(2):219-27. [PubMed: 25683772]

27. Hájek R, et al. A randomized phase III study of carfilzomib vs low-dose corticosteroids with optional cyclophosphamide in relapsed and refractory multiple myeloma (FOCUS). Leukemia. 2017; 31(1):107-114. [PubMed: 27416912]

28. Watanabe T, et al. A phase $1 / 2$ study of carfilzomib in Japanese patients with relapsed and/or refractory multiple myeloma. British Journal of Haematology. 2016; 172(5):745-756. [PubMed: 26732066] 
29. Berdeja JG, et al. A phase I/II study of the combination of panobinostat (PAN) and carfilzomib (CFZ) in patients (PTS) with relapsed or relapsed/refractory multiple myeloma (MM): Comparison of two expansion cohorts. Blood. 2015; 126(23):1825.

30. Berenson JR, et al. Replacement of bortezomib with carfilzomib for multiple myeloma patients progressing from bortezomib combination therapy. Leukemia. 2014; 28(7):1529-1536. [PubMed: 24429497]

31. Lendvai N, et al. A phase 2 single-center study of carfilzomib $56 \mathrm{mg} / \mathrm{m}^{2}$ with or without low-dose dexamethasone in relapsed multiple myeloma. Blood. 2014; 124(6):899-906. [PubMed: 24963043]

32. Dimopoulos MA, et al. Carfilzomib and dexamethasone versus bortezomib and dexamethasone for patients with relapsed or refractory multiple myeloma (ENDEAVOR): And randomised, phase 3, open-label, multicentre study. The Lancet Oncology. 2016; 17(1):27-38. [PubMed: 26671818]

33. Moreau P, et al. Impact of prior treatment on patients with relapsed multiple myeloma treated with carfilzomib and dexamethasone vs bortezomib and dexamethasone in a subgroup analysis of the phase 3 endeavor study (NCT01568866). Blood. 2015; 126(23):729.

34. Chng WJ, et al. Efficacy and safety of carfilzomib and dexamethasone vs bortezomib and dexamethasone in patients with relapsed multiple myeloma based on cytogenetic risk status: Subgroup analysis from the phase 3 study endeavor (NCT01568866). Blood. 2015; 126(23):30.

35. Ludwig H, et al. Carfilzomib and dexamethasone vs bortezomib and dexamethasone in patients with relapsed multiple myeloma: results of the phase 3 study ENDEAVOR (NCT01568866) according to age subgroup. Leuk Lymphoma. 2017:1-4.

36. Stewart AK, et al. Carfilzomib, lenalidomide, and dexamethasone vs lenalidomide and dexamethasone in patients (Pts) with relapsed multiple myeloma: Interim results from ASPIRE, a randomized, open-label, multicenter phase 3 study. Blood. 2014; 124(21)

37. Moreau $\mathrm{P}$, et al. A randomized, multicenter, phase (Ph) III study comparing carfilzomib (CFZ), lenalidomide (LEN), and dexamethasone (Dex) to LEN and Dex in patients (Pts) with relapsed multiple myeloma (MM). Journal of Clinical Oncology. 2011; 29(15)

38. Siegel DS, et al. A phase 2 study of single-agent carfilzomib (PX-171-003-A1) in patients with relapsed and refractory multiple myeloma. Blood. 2012; 120(14):2817-25. [PubMed: 22833546]

39. Stewart AK, et al. Carfilzomib, lenalidomide, and dexamethasone for relapsed multiple myeloma. N Engl J Med. 2015; 372(2):142-52. [PubMed: 25482145]

40. Vij R, et al. An open-label, single-arm, phase 2 study of single-agent carfilzomib in patients with relapsed and/or refractory multiple myeloma who have been previously treated with bortezomib. Br J Haematol. 2012; 158(6):739-48. [PubMed: 22845873]

41. Kaufman JL. Phase I Study of the Combination of Carfilzomib and Panobinostat for Patients with Relapsed and Refractory Myeloma: A Multiple Myeloma Research Consortium (MMRC) Clinical Trial. 2014

42. Bringhen S, et al. Carfilzomib, cyclophosphamide, and dexamethasone in patients with newly diagnosed multiple myeloma: a multicenter, phase 2 study. Blood. 2014; 124(1):63-9. [PubMed: 24855212]

43. Vesole DH, et al. Phase I study of carfilzomib, lenalidomide, vorinostat, and dexamethasone in patients with relapsed and/or refractory multiple myeloma. Br J Haematol. 2015; 171(1):52-9. [PubMed: 26018491]

44. Dimopoulos MA, et al. Carfilzomib and dexamethasone versus bortezomib and dexamethasone for patients with relapsed or refractory multiple myeloma (ENDEAVOR): a randomised, phase 3, open-label, multicentre study. Lancet Oncol. 2016; 17(1):27-38. [PubMed: 26671818]

45. Morawska M, et al. Therapy-related peripheral neuropathy in multiple myeloma patients. Hematol Oncol. 2015; 33(4):113-9.

46. Schlafer D, et al. Safety of proteasome inhibitors for treatment of multiple myeloma. Expert Opinion on Drug Safety. 2017; 16(2):167-183. [PubMed: 27841029]

47. Richardson PG, et al. Lenalidomide, bortezomib, and dexamethasone combination therapy in patients with newly diagnosed multiple myeloma. Blood. 2010; 116(5):679-86. [PubMed: 20385792] 
48. Jakubowiak AJ. Daratumumab (DARA) in combination with carflzomib, lenalidomide, and dexamethasone (KRd) in patients (pts) with newly diagnosed multiple myeloma (MMY1001): An open-label, phase 1b study. Jun.2017

49. Bringhen S, et al. A multicenter, open label phase I/II study of carfilzomib, pomalidomide and dexamethasone in relapsed and/or refractory multiple myeloma (MM) patients. Blood. 2016:128. Conference: 58th annual meeting of the american society of hematology, ASH 2016. United states. Conference start: 20161203. Conference end: 20161206. [PubMed: 27827828]

50. Papadopoulos KP, et al. A phase $1 \mathrm{~b} / 2$ study of prolonged infusion carfilzomib in patients with relapsed and/or refractory (R/R) multiple myeloma: Updated efficacy and tolerability from the completed 20/56mg/m2 expansion cohort of PX-171-007. Blood. 2011; 118(21)

51. ClinicalTrials.gov. A Study in Subjects With Relapsed and Refractory Multiple Myeloma Receiving Carfilzomib in Combination With Dexamethasone, Comparing Once-weekly Versus Twice-weekly Carfilzomib Dosing (ARROW). Apr.2017 
Data identification, screening, eligibility testing and inclusion according to PRISMA guidelines

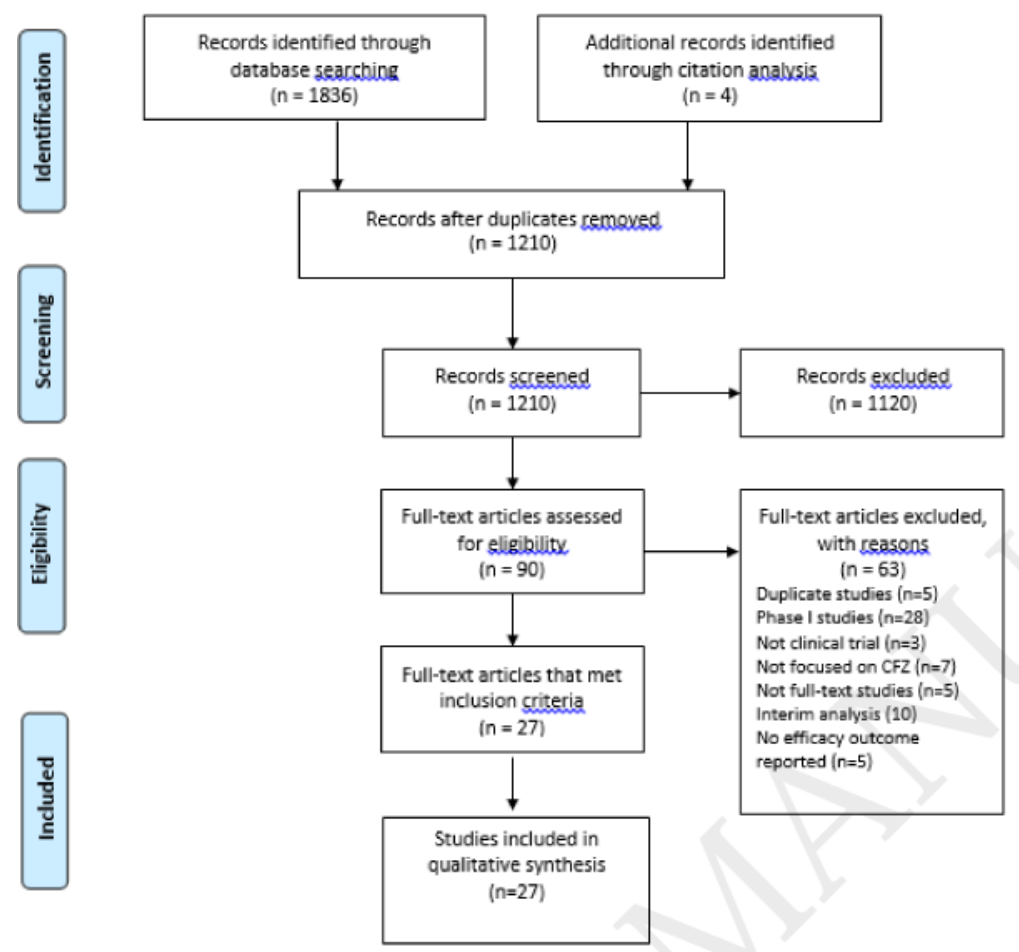

Fig 1.

Records identified through PubMed, EMBASE, Cochrane, Web of Science, Scopus, CINAHL and ClinicalTrials.gov database searches. Relevant articles from following conference proceedings were also included: the European Hematology Association, the American Society of Hematology, the American Society of Oncology and the American Society of Bone Marrow Transplantation. 


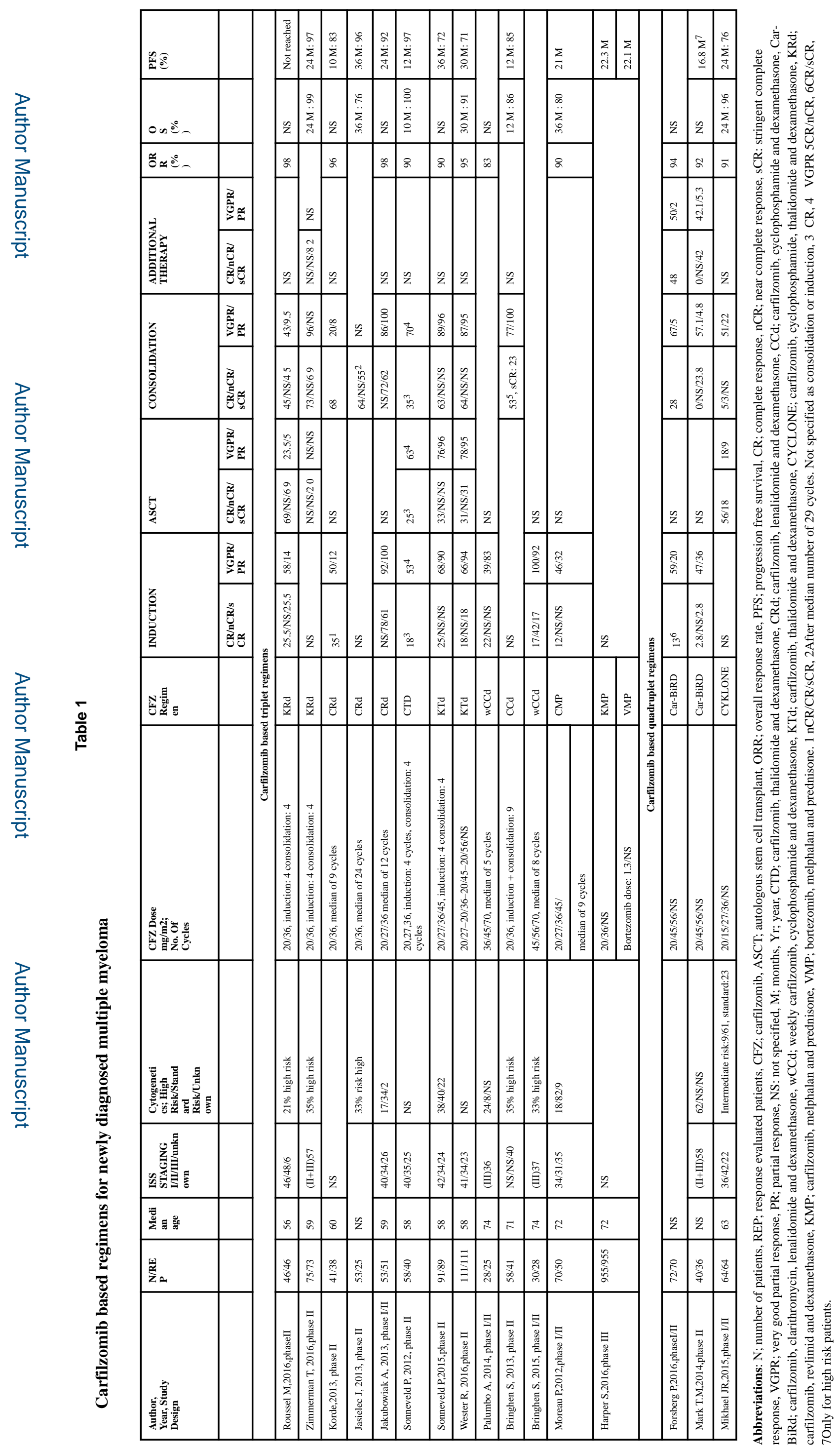




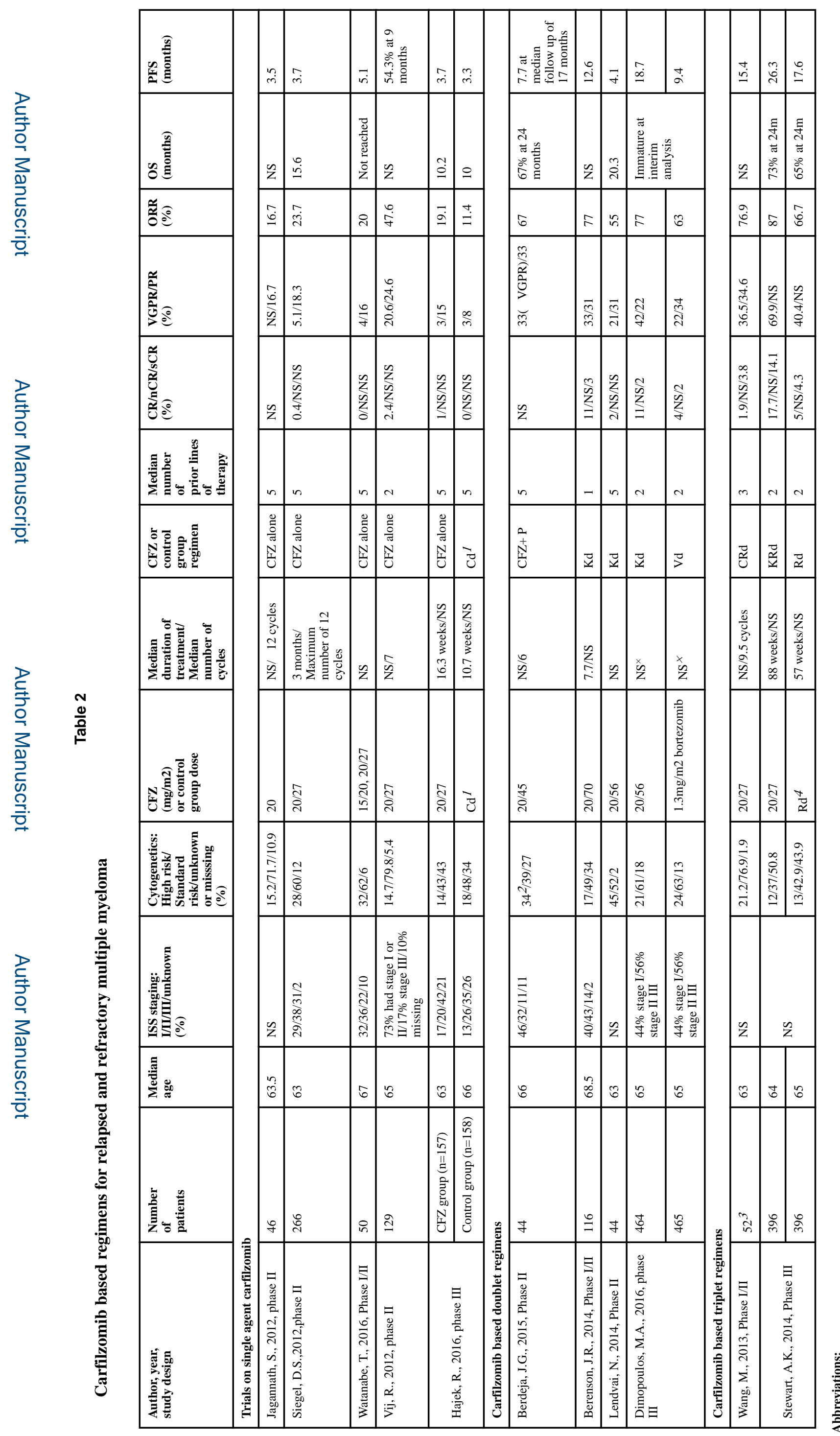




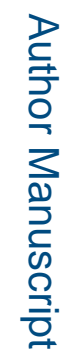

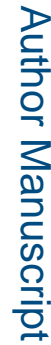

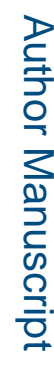

要

Crit Rev Oncol Hematol. Author manuscript; available in PMC 2019 May 01 